\title{
FRECUENCIAS Y PERIODOS PREDOMINANTES DE SISMOS REGISTRADOS EN LA CIUDAD DE TACNA, MEDIANTE ANÁLISIS Y ESPECTRO DE FOURIER
}

\author{
FREQUENCIES AND PREDOMINANT PERIODS OF EARTHQUAKES \\ REGISTERED IN THE CITY OF TACNA, BY MEANS OF ANALYSIS AND \\ FOURIER SPECTRUM
}

Wilber Mendoza Ramirez ${ }^{1}$ Dina Marlene Cotrado Flores ${ }^{2}$

Joel Ticahuanca Mamani ${ }^{3}$

Arleth Mendoza Chura ${ }^{4}$

Información del artículo:

Recibido: 03/12/2019

Aceptado: 10/06/2020

\footnotetext{
${ }^{1}$ Docente de la Facultad de Ingeniería Civil, Arquitectura, Geología Geotecnia de la Universidad Nacional Jorge Basadre Grohmann.

${ }^{2}$ Docente de la Facultad de Ingeniería de la Universidad Privada de Tacna.

${ }^{3}$ Estudiante de escuela Ingeniería Civil de la Universidad Privada de Tacna. ${ }^{4}$ Estudiante de escuela Geología Geotecnia la Universidad Nacional Jorge Basadre Grohmann. E-mail: ${ }^{1}$ wmendozar@unjbg.edu.pe, ${ }^{2}$ dcotrado@upt.edu.pe, ${ }^{3}$ joeticm@gmail.com, ${ }^{4}$ arlethmc@unjbg.edu.pe
} 


\section{Resumen}

La ciudad de Tacna se encuentra en silencio sísmico, esperando un sismo de gran magnitud y los efectos medioambientales que éste ocasione. El objetivo de la investigación fue determinar las frecuencias y periodos predominantes de algunos sismos registrados en la ciudad de Tacna, mediante el análisis y espectro de Fourier. Se seleccionó sismos del presente siglo, de magnitud mayor a 5,0 Mw y de diferentes fuentes sismogénicas cercanas, intermedias y lejanas. La investigación empleo el modelo matemático de Fourier, para procesar las señales sísmicas, del dominio del tiempo al dominio de la frecuencia, luego, se analizó y conoció las frecuencias y periodos predominantes de las diferentes señales. Para un mejor procesamiento de señales, se creó un código en Matlab para realizar los cálculos, que luego fueron verificados mediante el software Seismosignal. Las frecuencias predominantes de los sismos de fuente cercana, varían de 2,12 a 11,43 Hz (periodos predominantes de 0,09 a $0,47 \mathrm{~s}$ ); sismos de fuente intermedia, varían de 5,15 a $11,29 \mathrm{~Hz}$ (periodos predominantes de 0,09 a 0,19 s); y sismos de fuente lejana, varían de 0,43 a $6,06 \mathrm{~Hz}$ (periodos predominantes de 0,17 a 2,34 s). Al tener la mayoría de los sismos periodos predominantes cortos, estos pudieron generar fenómenos de resonancia a estructuras con periodos de vibración menores a 0,5 s.

Palabras clave: Dominio de la frecuencia; frecuencia predominante; fuentes sismogénicas; resonancia.

\section{Abstract}

The city of Tacna is in seismic silence, waiting for a large earthquake and the environmental effects that this causes. The objective of the investigation was to determine the prevailing frequencies and periods of some earthquakes registered in the city of Tacna, using the Fourier analysis and spectrum. Earthquakes of the present century, of a magnitude greater than $5.0 \mathrm{Mw}$ and from different near, intermediate and distant seismogenic sources were selected. The research used the Fourier mathematical model to process seismic signals, from the time domain to the frequency domain, then, the prevailing frequencies and periods of the different signals were analyzed and learned. For better signal processing, a code was created in Matlab to perform the calculations, which were then verified using the Seismosignal software. The predominant frequencies of earthquakes from close sources vary from 2.12 to $11.43 \mathrm{~Hz}$ (predominant periods from 0.09 to $0.47 \mathrm{~s}$ ); earthquakes of intermediate source, vary from 5.15 to $11.29 \mathrm{~Hz}$ (predominant periods from 0.09 to $0.19 \mathrm{~s}$ ); and earthquakes from distant sources, vary from 0.43 to $6.06 \mathrm{~Hz}$ (predominant periods from 0.17 to $2.34 \mathrm{~s}$ ). As most of the earthquakes had predominantly short periods, these could generate resonance phenomena to structures with periods of vibration less than $0.5 \mathrm{~s}$.

Keywords: Frequency domain; prevailing frequency; seismogenic sources; resonance. 


\section{Introducción}

La Tierra se puede idealizar como un sistema dinámico. Esto se debe a la interacción de las fuerzas geológicas del interior de la Tierra. La corteza terrestre está constituida por las llamadas placas tectónicas, las cuales, en su interacción, producen los sismos y volcanes. El Cinturón Sísmico del Pacífico es la principal zona de peligro sísmico mundial y se extiende a lo largo de la costa occidental de América, casi todas las zonas de subducción presentes en la Tierra se ubican alrededor del océano Pacífico (Jiménez, 2007), en la cual se encuentra la ciudad de Tacna.

La región sur occidental del Perú está situada en la zona de subducción de la placa Nazca y la placa Sudamericana. Ésta es una zona de alta actividad sísmica en donde, de acuerdo a la sismicidad histórica, han ocurrido sismos severos, entre los que tenemos: El sismo de 1604, el sismo de 1687, el sismo de 1784, el sismo de 1868. El periodo de recurrencia de estos sismos severos es del orden de un siglo, por lo que esta región es considerada de alto peligro sísmico ante la posibilidad de ocurrencia de un gran evento en el futuro cercano (Olarte y López, 2001)

Una forma de visualizar los efectos medioambientales de los sismos, es mediante el colapso de estructuras. Éstas son vulnerables al contenido frecuencial de los sismos, siendo el caso más desfavorable aquel que causa efectos de resonancia en las estructuras, resulta indiscutible la necesidad del análisis de los registros acelerográficos (Pérez, 2009). Del análisis de acelerogramas, además de la aceleración máxima del suelo y la duración, se obtiene información de la frecuencia predominante, que es una de las características de los sismos con mayor aplicación en el diseño sismorresistente de edificaciones (Naeim, 1989).

\section{Objetivos}

El objetivo de la presente investigación fue determinar las frecuencias y periodos predominantes de algunos sismos registrados en la ciudad de Tacna, mediante el análisis y espectro de Fourier, llevar un acelerograma del dominio del tiempo al dominio de la frecuencia, graficar un espectro de frecuencias e identificar los valores picos.

\section{Metodología}

El método utilizado es el deductivo. La orientación es básica. El enfoque es cuantitativo, debido a que los datos son producto de mediciones, se representan mediante números, y para el análisis se deben utilizar procedimientos estandarizados y aceptados por una comunidad científica (Hernández, Fernández y Baptista, 2014, p.5). La recolección de datos es retrolectiva, debido a que los datos se obtienen de fuentes secundarias existentes, como la base de datos de El Centro Peruano Japonés de Investigaciones Sísmicas y Mitigación de Desastres CISMID y La Red Acelerográfica UPGFIC-UNI.

\subsection{Selección de acelerogramas}

Se seleccionaron 10 eventos sísmicos ocurridos en los últimos 20 años, en la zona sur del Perú. El primer criterio de selección fue que las amplitudes sean mayores a $5.0 \mathrm{Mw}$. Desde el punto de vista cuantitativo, la magnitud, que es una medida de la energía que se libera en la fuente, a mayor magnitud en la fuente se presentan proporcionalmente mayores aceleraciones. El segundo criterio, es la proximidad de la fuente sismogénica. Fuente cercana, eventos a distancias epicentrales 
menores a $50 \mathrm{~km}$; fuente intermedia, eventos a distancias epicentrales entre 50 y $100 \mathrm{~km}$; y fuente lejana, distancias epicentrales mayores a $100 \mathrm{~km}$ (Chaparro, 2017).

En la Figura 1 se observa que el sismo del 23 de junio del 2001 tuvo su epicentro muy alejado de la ciudad de Tacna, pero tal fue su magnitud que ocasiono el colapso de algunas edificaciones.

\section{Figura 1}

Ubicación de sismos analizados, según fuente sismogénica, entre los años 2000 y 2020

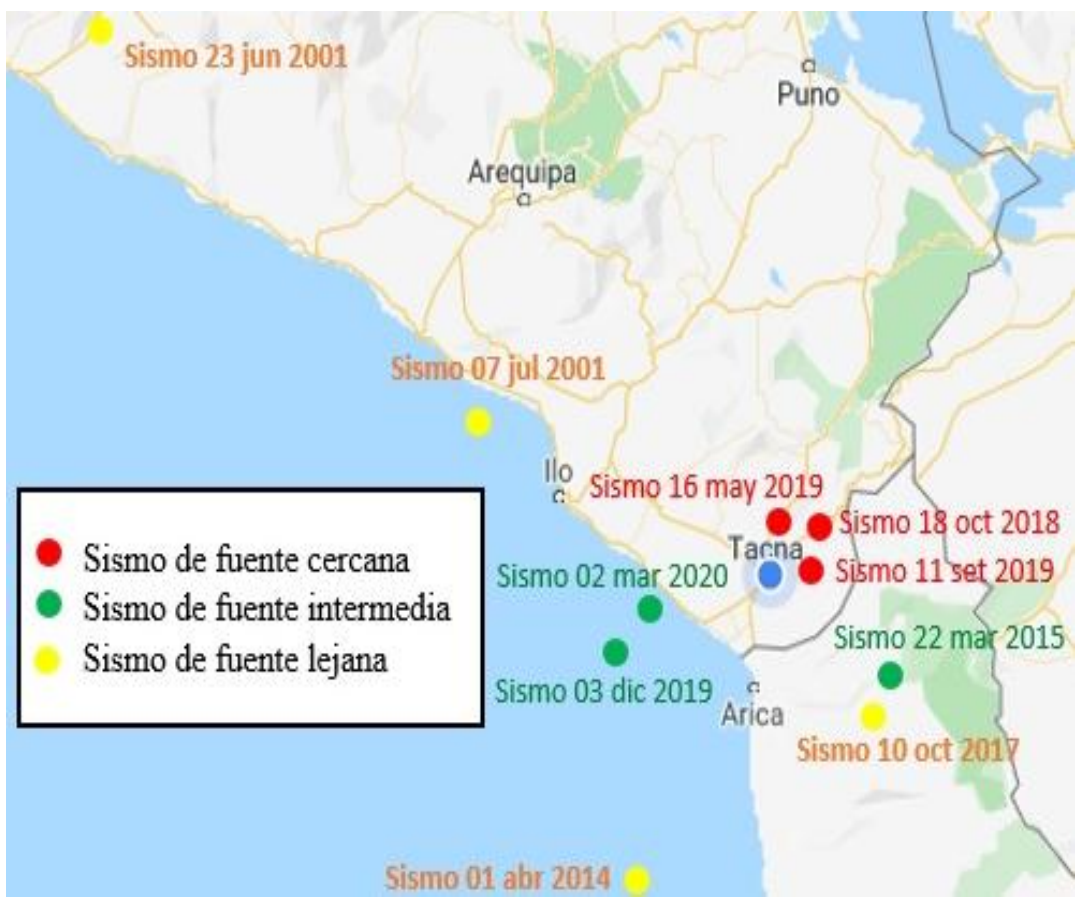

Nota. La figura representa la ubicación del epicentro de los sismos analizados, los cuales fueros registrados por estaciones ubicadas en la región Tacna.

Los detalles de los eventos sísmicos y las estaciones donde fueron medidas, se muestran en la Tabla 1, donde se puede destacar dos sismos de mayor magnitud, el del 23 de junio del 2001 y el sismo del 1 de abril del 2014, ambos con 8,2 Mw. La representación gráfica de los 10 acelerogramas se pueden observar en la Figura 2, donde claramente se visualiza que los sismos de fuente cercana e intermedia, tuvieron una similitud en su comportamiento vibratorio. 
Tabla 1

Información de sismos analizados con magnitudes superiores a 5,0 Mw, registrados en Tacna

\begin{tabular}{|c|c|c|c|c|c|c|c|c|}
\hline \multicolumn{3}{|c|}{ Información del sismo } & \multicolumn{6}{|c|}{ Información de estación } \\
\hline \multirow{2}{*}{ Fecha } & \multirow{2}{*}{$\begin{array}{l}\text { Magmitud } \\
(\mathrm{Mw})\end{array}$} & \multirow{2}{*}{$\begin{array}{l}\text { Profundidad } \\
\text { (km) }\end{array}$} & \multirow{2}{*}{ Código estación } & \multicolumn{2}{|c|}{ PGA $\left(\mathrm{cm} / \mathrm{s}^{2}\right)$} & \multirow{2}{*}{$\begin{array}{c}\text { Distancia } \\
\text { epicentro } \\
(\mathrm{km}) \\
\end{array}$} & \multirow{2}{*}{$\begin{array}{c}\text { Distancia } \\
\text { hipocentro } \\
(\mathbf{k m})\end{array}$} & \multirow{2}{*}{$\begin{array}{c}\text { Fuente } \\
\text { sismogénica }\end{array}$} \\
\hline & & & & EW & NS & & & \\
\hline 18-Oct-18 & 5,0 & 102,00 & C1EA-TAC-CR & 11,73 & \multirow{6}{*}{6.27} & 28,01 & 105,78 & \multirow{3}{*}{$\begin{array}{l}\text { Fuente } \\
\text { cercana }\end{array}$} \\
\hline 16-May-19 & 5,1 & 103,00 & C189 TAC-AA & 28,15 & & 12,36 & 103,74 & \\
\hline 11-set.19 & 5,6 & 104,00 & C1EA-TAC-CR & & & 19,34 & 105,78 & \\
\hline 22-Mar-15 & 7,4 & 125,00 & TAC002 & 34,50 & & 97,42 & 158,23 & \multirow{3}{*}{$\begin{array}{c}\text { Fuente } \\
\text { intermedia }\end{array}$} \\
\hline 3-Dic-19 & 6,0 & 40,00 & C189 TAC-AA & 37,73 & & 83,25 & 92,36 & \\
\hline 2-Mar-20 & 5,0 & 25,00 & C1EA-TAC-CR & 20,95 & & 54,30 & 59,78 & \\
\hline 23-Jun-01 & 8,2 & 33,00 & MOQ001 & 295,15 & \multirow{3}{*}{40.42} & 327,17 & 359,88 & \multirow{4}{*}{$\begin{array}{l}\text { Fuente } \\
\text { lejana }\end{array}$} \\
\hline 7-Jul-01 & 7,5 & 33,00 & TAC001 & & & 165,92 & 169,08 & \\
\hline 1-Abr-14 & 8,2 & 38,90 & TAC001 & 72,49 & & 185,66 & 188,81 & \\
\hline 10-Oct-17 & 7,6 & 100,00 & C16B-TAC-CA & 63,85 & & 111,25 & 149.59 & \\
\hline
\end{tabular}

Nota. Esta tabla, PGA es la aceleración máxima del registro de aceleración, EW componente este oeste, NS componente norte sur de las aceleraciones registradas.

\section{Figura 2}

Representación gráfica de acelerogramas analizados con magnitudes superiores a $5.0 \mathrm{Mw}$
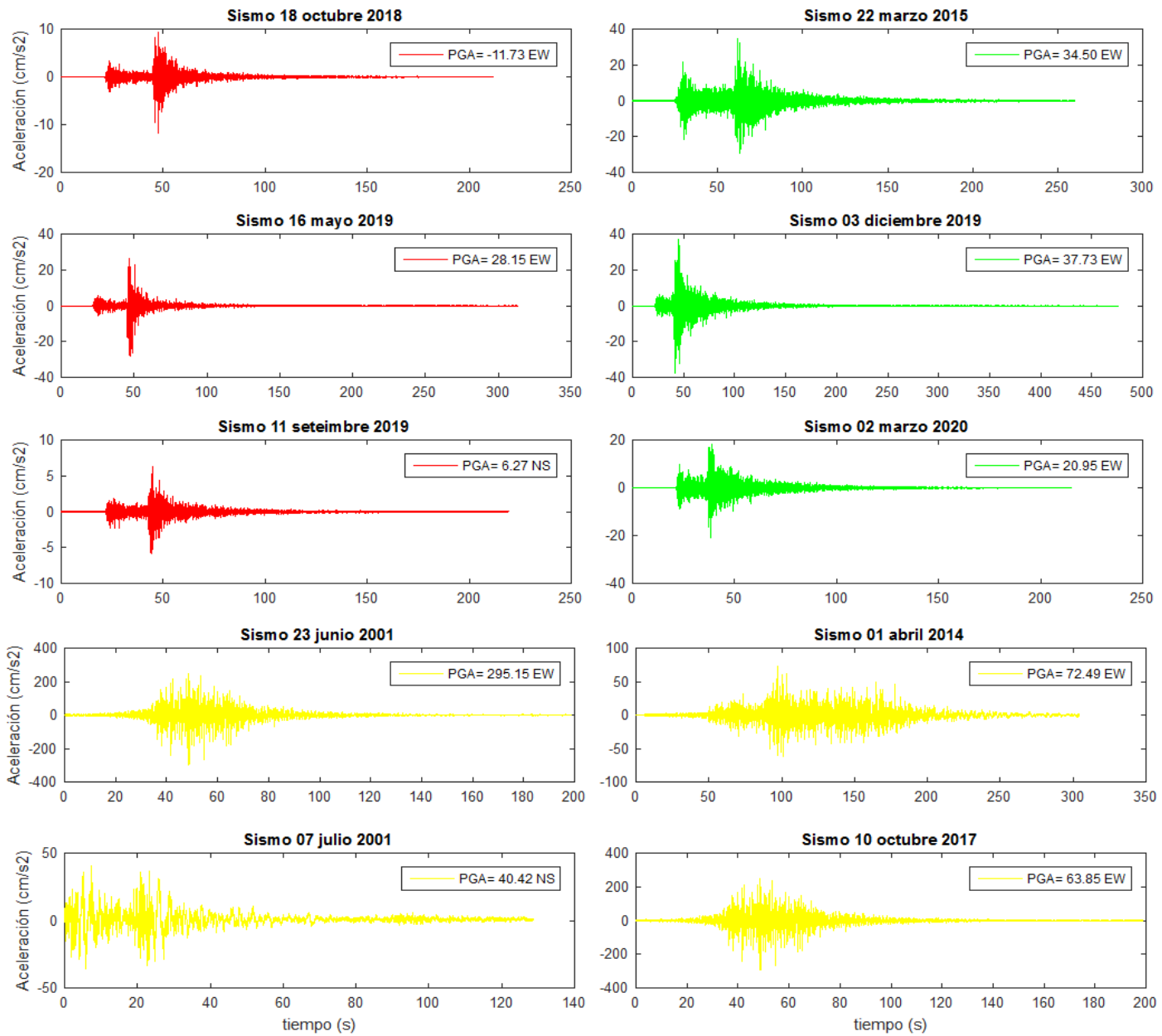

Nota. En la figura se destaca el sismo del 23 de junio 2001, por tener un PGA elevado de $295.15 \mathrm{~m} / \mathrm{s}^{2}$. 


\subsection{Herramientas matemáticas}

La transformada de Fourier es una potente herramienta de análisis matemático que se utiliza en diversos campos de la ciencia. En la presente investigación se emplea para transformar una señal analógica unidimensional del dominio del tiempo al dominio de la frecuencia (Jiménez, 2007, p.47).

Para el caso continuo la Transformada de Fourier $F(\omega)$ de la función continua $f(t)$, se define:

$$
F(\omega)=\int_{-\infty}^{\infty} f(t) \cdot e^{-j \omega t} \cdot d t
$$

Para el caso discreto, como los registros de aceleraciones, señal analógica con intervalos de tiempos constante, la Transformada Discreta de Fourier $A_{k}$ de una sucesión de $\mathrm{N}$ puntos, se define (James, 2011, p.644-683):

$$
A_{k}=\sum_{n=0}^{N-1} a_{n} \cdot e^{-j n \Delta \omega k T} ; k=0,1,2, \ldots
$$

Donde $A_{k}$ es el espectro en función de la frecuencia discreta, $a_{n}$ es la señal discreta en función del tiempo, e $e^{-j n k \Delta \omega T}$ es el fasor de sondeo discreto (forma compleja), j es en número imaginario, $T$ es el tiempo entre señales muestrales (periodo), $\Delta \omega k=2 \pi k / N T$ es la frecuencia angular $k$ para cada señal $\left(f_{k}=k / N T\right.$ es la frecuencia $k$ para cada señal en el dominio de la frecuencia.

Aplicando las equivalencias anteriores y la definición de función real con valores complejos (Duoandikoetxea, 2003, p.5-7), la $A_{k}$ se puede expresar:

$$
A_{k}=\sum_{n=0}^{N-1} a_{n} \cos \left(\frac{2 \pi k n}{N}\right)-j \sum_{n=0}^{N-1} a_{n} \operatorname{sen}\left(\frac{2 \pi k n}{N}\right)
$$

Toda expresión compleja, tiene una parte real $\operatorname{Re}\left(A_{k}\right)$ y parte imaginaria $\operatorname{Im}\left(A_{k}\right)$ :

$$
\begin{aligned}
& \operatorname{Re}\left(A_{k}\right)=\sum_{n=0}^{N-1} a_{n} \cos \left(\frac{2 \pi k n}{N}\right) \\
& \operatorname{Im}\left(A_{k}\right)=\sum_{n=0}^{N-1} a_{n} \operatorname{sen}\left(\frac{2 \pi k n}{N}\right)
\end{aligned}
$$

A la vez se pueden representar en un plano llamado diagrama de Argand, como se muestra en la Figura 3.

La amplitud de la transformada discreta de Fourier $\left|A_{k}\right|$ y el ángulo de fase $\Phi_{k}$ se determinan mediante la siguiente expresión:

$$
\begin{gathered}
\left|A_{k}\right|=\sqrt{\left[\sum_{n=0}^{N-1} a_{n} \cos \left(\frac{2 \pi k n}{N}\right)\right]^{2}+\left[i \sum_{n=0}^{N-1} a_{n} \operatorname{sen}\left(\frac{2 \pi k n}{N}\right)\right]^{2}} \\
\Phi_{k}=\operatorname{atan}\left(\frac{\operatorname{Im}\left(A_{k}\right)}{\operatorname{Re}\left(A_{k}\right)}\right)
\end{gathered}
$$




\section{Figura 3}

Diagrama de Argand para expresión compleja

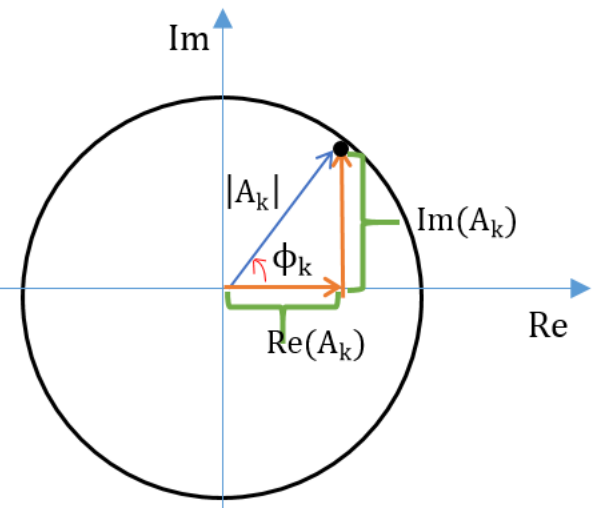

Nota. En la figura se muestra la representación de una expresión compleja, donde el eje de las abscisas es la parte real y eje de las ordenadas es la parte imaginaria.

\subsection{Procesamiento de señales}

Se aplicó el modelo matemático de Fourier, para analizar y conocer la frecuencia predominante de todos los sismos descritos en la Tabla 1, en particular se desarrolla el análisis del sismo del 23 de junio del 2001.

Sismo 23 de junio del 2001: Con una magnitud de 8,2 Mw. Acelerograma registrado en la estación sísmica MOQ001, ubicada a una latitud $-1,19$ y longitud $-70,93$, con una frecuencia de medición de $100 \mathrm{~Hz}$. El evento sísmico se produjo a una latitud -16,08 y longitud -73,77, a una distancia epicentral de $327,17 \mathrm{~km}$, y a una profundidad de $33 \mathrm{~km}$. El registro de aceleración se muestra en la Tabla 2.

\section{Tabla 2}

Registro de aceleración del sismo 23 de junio del 2001

\begin{tabular}{cc}
\hline Tiempot $(\mathrm{s})$ & $\begin{array}{c}\text { Aceleración } \mathrm{a}_{\mathrm{n}}\left(\mathrm{cm} / \mathrm{s}^{2}\right) \\
\text { EW }\end{array}$ \\
\hline 0,0000 & 0,0687 \\
0,0100 & 0,0691 \\
$\ldots$ & $\ldots$ \\
198,9000 & $-0,2905$ \\
198,9100 & 0,0004 \\
\hline
\end{tabular}

Nota. Acelerograma de componente este oeste EW, obtenido de la estación Moquegua, descargado de la página http://www.cismid.uni.edu.pe

La tabla 2 muestra datos iniciales y finales del registro de aceleración, debido a que el registro original tiene $\mathrm{N}=19892$ valores de aceleración registrados, en un intervalo de tiempo $\mathrm{T}=0,01$ s. 
Se realizó el procedimiento de cálculo de frecuencia y amplitud para dos valores iniciales y finales del registro total:

La frecuencia se determina con la siguiente expresión:

$$
\begin{gathered}
f_{k}=\frac{1}{N(T)} k \quad k=0,1,2, \ldots \\
f_{0}=\frac{1}{19892(0,01)}(0)=0 \mathrm{~Hz} \\
f_{1}=\frac{1}{19892(0.01)}(1)=0,0050 \mathrm{~Hz} \\
f_{19891}=\frac{1}{19892(0.01)}(19891)=99,9949 \mathrm{~Hz} \\
f_{19892}=\frac{1}{19892(0,01)}(19892)=100 \mathrm{~Hz}
\end{gathered}
$$

Para determinar la amplitud $\left|A_{k}\right|$, primero se debe conocer la parte real y parte imaginaria, aplicando la ecuación 4:

Para $k=0$

$$
\begin{gathered}
\operatorname{Re}\left(\mathrm{A}_{0}\right)=\sum_{\mathrm{n}=0}^{\mathrm{N}-1} \mathrm{a}_{\mathrm{n}} \cos \left(\frac{2 \pi(0) \mathrm{n}}{\mathrm{N}}\right) \\
(0,0687) \cos \left(\frac{2 \pi(0)(0)}{19892}\right)+(0,0691) \cos \left(\frac{2 \pi(0)(1)}{19892}\right)+(0.0712) \cos \left(\frac{2 \pi(0)(2)}{19892}\right)+\cdots \\
+(-0,2905) \cos \left(\frac{2 \pi(0)(19890)}{19892}\right)+(0,0004) \cos \left(\frac{2 \pi(0)(19891)}{19892}\right)=1399,3845 \\
\operatorname{Im}\left(\mathrm{A}_{0}\right)=\sum_{\mathrm{n}=0}^{\mathrm{N}-1} \mathrm{a}_{\mathrm{n}} \operatorname{sen}\left(\frac{2 \pi(0) \mathrm{n}}{\mathrm{N}}\right) \\
(0,0687) \operatorname{sen}\left(\frac{2 \pi(0)(0)}{19892}\right)+(0,0691) \operatorname{sen}\left(\frac{2 \pi(0)(1)}{19892}\right)+(0,0712) \operatorname{sen}\left(\frac{2 \pi(0)(2)}{19892}\right)+\cdots \\
+(-0,2905) \operatorname{sen}\left(\frac{2 \pi(0)(19890)}{19892}\right)+(0,0004) \operatorname{sen}\left(\frac{2 \pi(0)(19891)}{19892}\right)=0
\end{gathered}
$$

Luego, se emplea la ecuación 5:

$$
\left|A_{0}\right|=\sqrt{[1399.3845]^{2}+[0]^{2}}=1399.3845
$$

Para $k=1$

$$
\operatorname{Re}\left(A_{1}\right)=\sum_{n=0}^{N-1} a_{n} \cos \left(\frac{2 \pi(1) n}{N}\right)
$$


$(0,0687) \cos \left(\frac{2 \pi(1)(0)}{19892}\right)+(0.0691) \cos \left(\frac{2 \pi(1)(1)}{19892}\right)+(0.0712) \cos \left(\frac{2 \pi(1)(2)}{19892}\right)+\cdots$

$$
+(-0,2905) \cos \left(\frac{2 \pi(1)(19890)}{19892}\right)+(0,0004) \cos \left(\frac{2 \pi(1)(19891)}{19892}\right)=-10,95180
$$

$$
\operatorname{Im}\left(A_{1}\right)=\sum_{n=0}^{N-1} a_{n} \operatorname{sen}\left(\frac{2 \pi(1) n}{N}\right)
$$

$(0,0687) \operatorname{sen}\left(\frac{2 \pi(1)(0)}{19892}\right)+(0,0691) \operatorname{sen}\left(\frac{2 \pi(1)(1)}{19892}\right)+(0.0712) \operatorname{sen}\left(\frac{2 \pi(1)(2)}{19892}\right)+\cdots$

$$
+(-0,2905) \operatorname{sen}\left(\frac{2 \pi(1)(19890)}{19892}\right)+(0,0004) \operatorname{sen}\left(\frac{2 \pi(1)(19891)}{19892}\right)=-0,5476
$$

$$
\left|A_{1}\right|=\sqrt{[-10.95180]^{2}+[-0.5476]^{2}}=10,9655
$$

Para k=19891

$$
\begin{gathered}
\operatorname{Re}\left(A_{19891}\right)=\sum_{n=0}^{N-1} a_{n} \cos \left(\frac{2 \pi(19891) n}{N}\right) \\
(0,0687) \cos \left(\frac{2 \pi(19891)(0)}{19892}\right)+(0.0691) \cos \left(\frac{2 \pi(19891)(1)}{19892}\right)+(0.0712) \cos \left(\frac{2 \pi(19891)(2)}{19892}\right)+\cdots \\
+(-0,2905) \cos \left(\frac{2 \pi(19891)(19890)}{19892}\right) \\
+(0,0004) \cos \left(\frac{2 \pi(19891)(19891)}{19892}\right)=-10,9518 \\
\quad \operatorname{Im}\left(A_{19891}\right)=\sum_{n=0}^{N-1} a_{n} \operatorname{sen}\left(\frac{2 \pi(19891) n}{N}\right) \\
(0,0687) \sin \left(\frac{2 \pi(19891)(0)}{19892}\right)+(0,0691) \operatorname{sen}\left(\frac{2 \pi(19891)(1)}{19892}\right)+(0,0712) \operatorname{sen}\left(\frac{2 \pi(19891)(2)}{19892}\right)+\cdots \\
+(-0,2905) \operatorname{sen}\left(\frac{2 \pi(19891)(19890)}{19892}\right)+(0,0004) \operatorname{sen}\left(\frac{2 \pi(19891)(19891)}{19892}\right)=0.5476 \\
\left|A_{19891}\right|=\sqrt{[-10,95180]^{2}+[-0,5476]^{2}}=10,9655
\end{gathered}
$$

Para k=19892

$$
\operatorname{Re}\left(\mathrm{A}_{19892}\right)=\sum_{\mathrm{n}=0}^{\mathrm{N}-1} \mathrm{a}_{\mathrm{n}} \cos \left(\frac{2 \pi(19892) \mathrm{n}}{\mathrm{N}}\right)
$$




$$
\begin{aligned}
& \begin{array}{l}
(0,0687) \cos \left(\frac{2 \pi(19892)(0)}{19892}\right)+(0,0691) \cos \left(\frac{2 \pi(19892)(1)}{19892}\right)+(0,0712) \cos \left(\frac{2 \pi(19892)(2)}{19892}\right)+\cdots \\
+(-0,2905) \cos \left(\frac{2 \pi(19892)(19890)}{19892}\right)+(0,0004) \cos \left(\frac{2 \pi(19892)(19891)}{19892}\right) \\
=1399.3845
\end{array} \\
& \qquad \begin{array}{l}
\operatorname{Im}\left(\mathrm{A}_{19892}\right)=\sum_{\mathrm{n}=0}^{\mathrm{N}-1} \mathrm{a}_{\mathrm{n}} \operatorname{sen}\left(\frac{2 \pi(19892) \mathrm{n}}{\mathrm{N}}\right) \\
(0,0687) \operatorname{sen}\left(\frac{2 \pi(19892)(0)}{19892}\right)+(0.0691) \operatorname{sen}\left(\frac{2 \pi(19892)(1)}{19892}\right)+(0,0712) \operatorname{sen}\left(\frac{2 \pi(19892)(2)}{19892}\right)+\cdots \\
+(-0,2905) \operatorname{sen}\left(\frac{2 \pi(19892)(19890)}{19892}\right)=0 \\
\left|A_{19892}\right|=\sqrt{[1399.3845]^{2}+[0]^{2}}=1399,3845
\end{array}
\end{aligned}
$$

Los cálculos realizados, se puede resumir en la siguiente Tabla 3:

Tabla 3

Sismo del 23 de junio del 2001, en función del dominio de la frecuencia

\begin{tabular}{cc}
\hline $\mathbf{f}_{\mathbf{k}}(\mathbf{H z})$ & $\left|\mathbf{A}_{\mathbf{k}}\right|$ \\
\hline 0,00 & 1399,3845 \\
0,005 & 10,9655 \\
$\ldots$ & $\ldots$ \\
99,9949 & 10,9655 \\
100,0 & 1399,3845 \\
\hline
\end{tabular}

Nota. En la tabla se muestra las frecuencias y amplitudes para dos valores iniciales y finales, de un total 19892 frecuencias.

En la Tabla 3, se observa que $\left|A_{0}\right|=\left|A_{19892}\right|$ y $\left|A_{1}\right|=\left|A_{19891}\right|$. Esto es una consecuencia del hecho de que las exponenciales complejas discretas que difieren por un múltiplo de $2 \pi$ son idénticas (Oppenheim y Willsky, 2004, p.211). Y debido a esta simetría, solo es necesario representarla al $50 \%$ de los datos procesados, como se observa en la Figura 4. 


\section{Figura 4}

Sismo 23 junio del 2001, dominio de la frecuencia

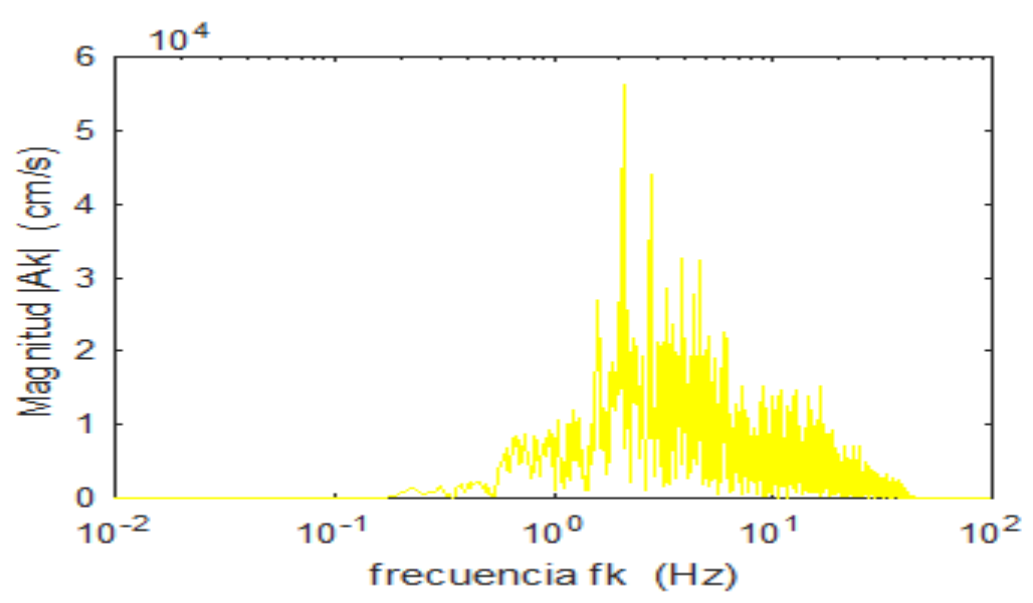

Nota. En la figura la abscisa de máxima amplitud de magnitud, es la frecuencia predominanate $f_{p}$ del acelerograma del sismo del 23 junio del 2001.

$$
f_{p}=1,05 \mathrm{~Hz}
$$

Y el periodo predominante es:

$$
T_{p}=\frac{1}{f_{p}}=0,96 s
$$

\section{Resultados}

Para un rápido análisis de los diferentes acelerogramas, se empleó el siguiente código en Matlab:

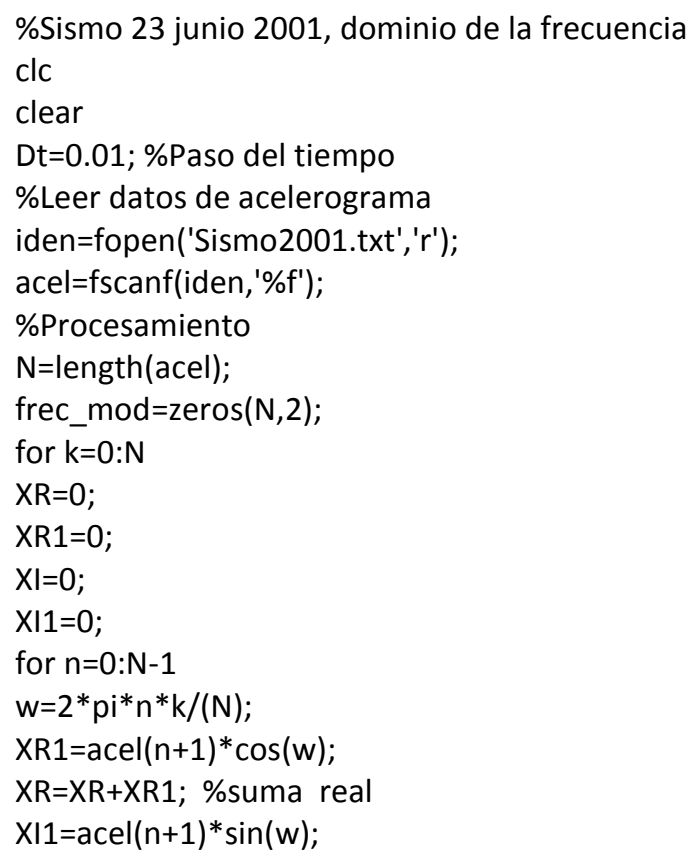


$\mathrm{XI}=\mathrm{XI}+\mathrm{XI}$ 1; \%suma imaginaria

end

amp $=\left(\left(X R^{\wedge} 2\right)+\left(X I^{\wedge} 2\right)\right)^{\wedge} 0.5$;

frec_mod $(k+1,2)=a m p$;

frec_mod $(k+1,1)=\left(1 /\left(D t^{*} N\right)\right) * k$;

end

frec_mod

\%Ploteo de gráfica

semilogx(frec_mod(1:N/2,1),frec_mod(1:N/2,2),'y')

xlabel('frecuencia fk $(\mathrm{Hz})$ ')

ylabel('Magnitud |Ak|')

Procesados las señales en el dominio de la frecuencia. En la Figura 5 se observa los sismos de fuente cercana; en la Figura 6 se observa los sismos de fuente intermedia; y en la Figura 7 se observa los sismos de fuente lejana.

\section{Figura 5}

Sismos de fuente cercana, dominio de la frecuencia.

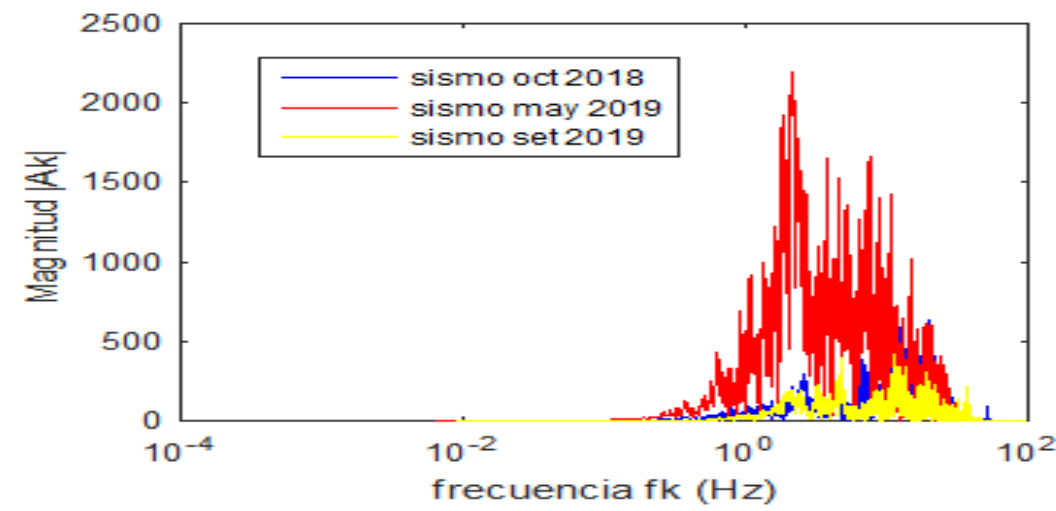

Nota. En la figura se presenta los 3 sismos analizados de fuente cercana en el dominio de la frecuencia, graficados con código Matlab.

\section{Figura 6}

Sismos de fuente intermedia, dominio de la frecuencia

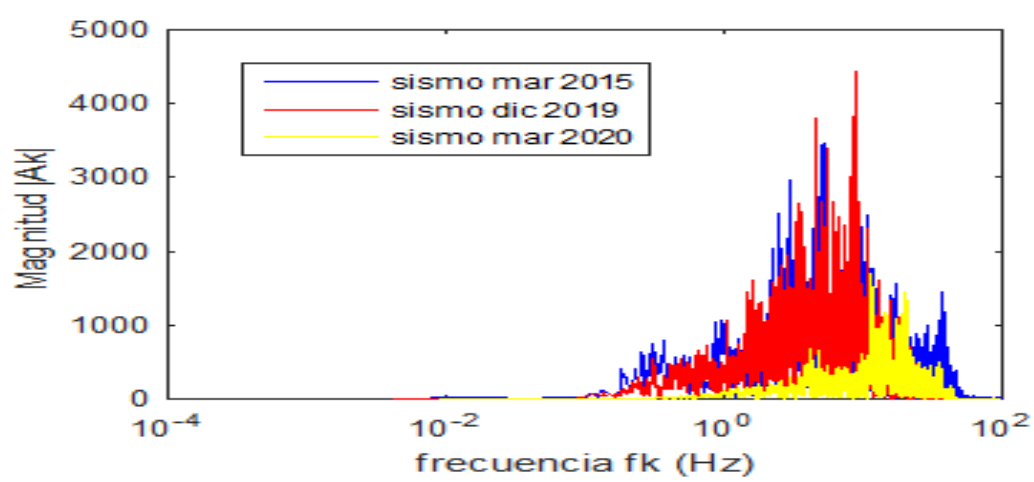

Nota. En la figura se presenta los 3 sismos analizados de fuente intermedia en el dominio de la frecuencia, graficados con código Matlab. 


\section{Figura 7}

Sismos de fuente lejana, dominio de la frecuencia.

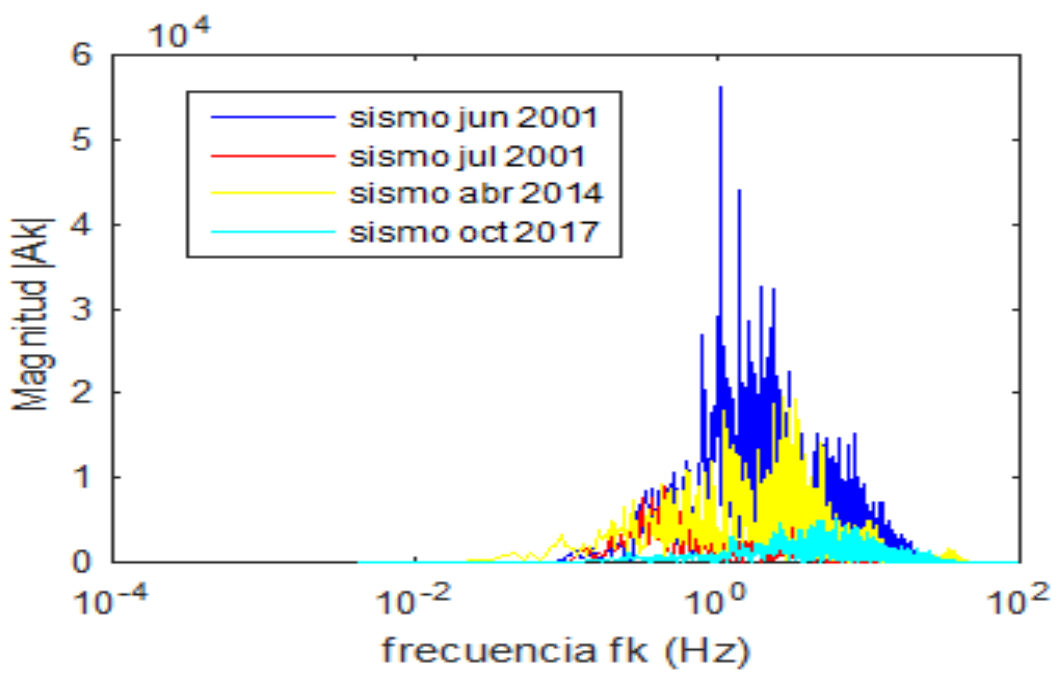

Nota. En la figura se presenta los 4 sismos analizados de fuente cercana en el dominio de la frecuencia, graficados con código Matlab.

Con los espectros de Fourier de cada acelerograma, se encontró las frecuencias y periodos predominantes, las cuales se muestran en la siguiente Tabla 4.

\section{Tabla 4}

Frecuencias y periodos predominantes de acelerogramas analizados

\begin{tabular}{|c|c|c|c|c|}
\hline Sismo & $\begin{array}{l}\text { Profundida } \\
\text { d (km) }\end{array}$ & $f_{p} \quad(H z)$ & $T_{p}$ & $\begin{array}{c}\text { Fuente } \\
\text { sismogénica }\end{array}$ \\
\hline 18-Oct-18 & 102,00 & 11,19 & 0,09 & \multirow{3}{*}{ Fuente cercana } \\
\hline 16-Мay-19 & 103,00 & 2,12 & 0,47 & \\
\hline 11-set.19 & 104,00 & 11,43 & 0,09 & \\
\hline 22-Mar-15 & 125,00 & 5,15 & 0,19 & \multirow{3}{*}{$\begin{array}{c}\text { Fuente } \\
\text { intermedia }\end{array}$} \\
\hline 3-Dic-19 & 40,00 & 8,99 & 0,11 & \\
\hline 2-Mar-20 & 25,00 & 11,29 & 0,09 & \\
\hline 23-Jun-01 & 33,00 & 1,05 & 0,96 & \multirow{4}{*}{ Fuente lejana } \\
\hline 7-Jul-01 & 33,00 & 0,43 & 2,34 & \\
\hline 1-Abr-14 & 38,90 & 2,65 & 0,38 & \\
\hline $10-O c t-17$ & 100,00 & 6,06 & 0,17 & \\
\hline
\end{tabular}

Nota. En la tabla se muestra frecuencias altas en la mayoría de los sismos analizados, con la excepción del sismo del 7 de julio del 2001.

En la Figura 8 Se traza una línea envolvente al espectro de frecuencias, para resaltar el incremento y decaimiento de amplitudes en rampa 


\section{Figura 8}

Decaimiento brusco de la frecuencia predominante de sismo del 11 de setiembre 2019.

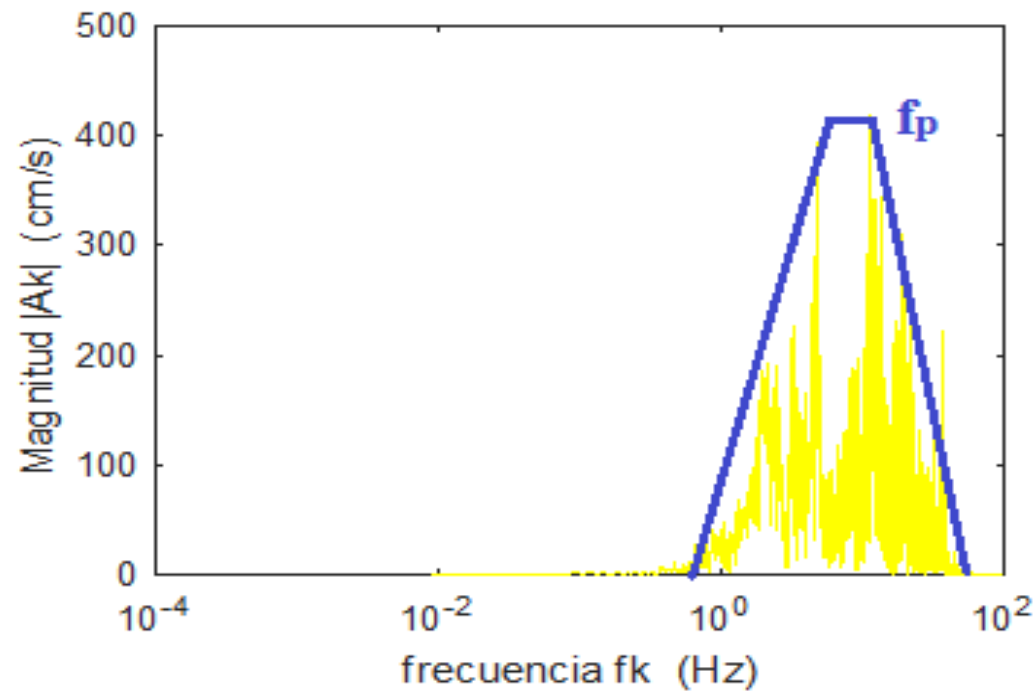

Nota.

En la figura se resalta la línea envolvente de las frecuencias del sismo, donde se puede apreciar mejor el decaimiento de la frecuencia predominante.

\section{Discusión}

Las frecuencias predominantes conocidas de los sismos procesados, son de alta frecuencia mayores a $1 \mathrm{~Hz}$ y varían de 1,05-11,43 Hz, con la salvedad del sismo del 7 julio 2001, que tiene una frecuencia de $0.43 \mathrm{~Hz}$. Nuestros sismos tienen altas frecuencias y desplazamientos bajos (Muñoz, 2018).

La mayoría de los sismos analizados, debieron afectar a edificaciones de baja altura, menores a 5 niveles, por el efecto de la resonancia, con la excepción de los sismos 23 de junio y 7 julio del 2001. El tiempo de duración del sismo es importante, ya que la amplificación de desplazamientos no se produce de manera inmediata. La frecuencia (o periodo) de resonancia se define como la frecuencia de excitación en la que el factor de amplificación sísmica de una estructura es máxima. La deformación vibratoria no se vuelve infinita de inmediato, sino poco a poco (Chopra, 2014, p.70-82).

Los espectros de Fourier de las Figuras 5, 6 y 7 presentan alta e intermedia absorción de la máxima frecuencia, esto se visualiza en el abrupto decaimiento de la frecuencia máxima, debido a que los eventos interplaca analizados tienen profundidades que los ubican en la estructura superior de contacto, con profundidades menores a $40 \mathrm{~km}$, y eventos interplaca profundos, con profundidades mayores a $100 \mathrm{~km}$. Este criterio se visualiza mejor en la Figura 8, donde se visualiza un decaimiento brusco de la frecuencia predominante. El estudio el efecto de la dispersión y absorción de alta frecuencia para las ondas sísmicas en la zona de subducción del norte de Chile. Encontrando 3 estructuras tectónicas diferentes que controlan el contenido de ondas sísmicas de alta frecuencia. La primera estructura corresponde a la parte más superior del contacto, se produce un abrupto decaimiento de la alta frecuencia en el espectro de Fourier. El segundo se señala que corresponde a una zona donde ocurren eventos interplaca más profundos y se puede observar una 
baja atenuación de ondas de alta frecuencia, finalmente para eventos intraplaca con profundidades mayores a $60 \mathrm{~km}$, poseen una atenuación intermedia (Espinoza, 2017, p.8-12).

Aunque no se analizaron una gran cantidad de acelerogramas, se podría generar en las Figuras 5, 6 y 7 una línea envolvente que caracterice los espectros de Fourier, de tal manera que nos proporcionen intervalos de frecuencias predominantes, según las fuentes analizadas. Gracias a la superposición de los espectros, es que los espectros de Fourier de los sismos de fuente intermedia, lejana y cercana se acoplan considerablemente, tanto en términos de frecuencia como en amplitud, aunque estos últimos son más pronunciados en picos espectrales (Pérez, Torres, Guada, 2012, p.11).

Mediante la transformada discreta de Fourier se transformó 10 acelerogramas, del dominio del tiempo al dominio de la frecuencia, con ayuda de un código en Matlab, el cual sus resultados fueron verificados con el software Seismosignal.

Los sismos analizados del sur del Perú son de frecuencias altas que varían de $1.05-11,43 \mathrm{~Hz}$. El sismo del 7 de julio del 2001 con frecuencia predominante de $0,43 \mathrm{~Hz}$, es un sismo raro, y debido a la cercanía de ocurrencia en tiempo del sismo del 23 de junio del 2001, se trate de una réplica de éste evento sísmico.

Las frecuencias predominantes de los sismos de fuente cercana, varían de 2,12 a $11,43 \mathrm{~Hz}$ (periodos predominantes de 0,09 a 0,47 s); sismos de fuente intermedia, varían de 5,15 a 11,29 Hz (periodos predominantes de 0.09 a 0.19 s); y sismos de fuente lejana, varían de 0,43 a 6,06 Hz (periodos predominantes de 0,17 a $2,34 \mathrm{~s}$ ).

La mayoría de los sismos analizados presentan periodos predominantes cortos, estos pudieron generar fenómenos de resonancia y hacer daño a estructuras con periodos de vibración menores a $0,5 \mathrm{~s}$.

\section{Referencias bibliográficas}

Chaparro, Angela. (2017). Selección y procesamiento de acelerogramas para el análisis dinámico de la presa Teatinos. Ingenio Magno, 8(2): 109-124. ISSN En Línea 2422-2399. Disponible en http://revistas.ustatunja.edu.co/index.php/ingeniomagno/article/view/1316

Chopra, Anil. (2014). Dinámica de estructuras. 4ạ ed. México: Pearson Educación, pp. 70-82. ISBN: 978-607-32-2239-6

Cismid. (15 de enero 2020). Universidad Nacional de Ingeniería FIC-UNI. Disponible en http://www.cismid.uni.edu.pe/

Duoandikoetxea, Javier. (2003). Lecciones sobre las series y transformadas de Fourier. Apuntes curso Análisis de Fourier en Maestría en Matemáticas, UNAN-Managua., pp. 5-7. Disponible en

ttps://www.ugr.es/ acanada/docencia/matematicas/analisisdefourier/Duoandikoetxeafouri er.pdf

Espinoza, Matías. (2017). Análisis de la demanda sísmica de diferentes tipos de sismos chilenos en base a la atenuación de alta frecuencia. Tesis de pregrado. Universidad de Chile. pp.8-12. Disponible en http://repositorio.uchile. cl/handle/2250/148027

Hernández, Roberto, FERNÁNDEZ, Carlos y BAPTISTA, Pilar. (2014). Metodología de la Investigación. $6^{a}$ ed., México: mexicana, 5 pp. ISBN: 978-1-4562-2396-0 
James, Glyn et al. (2011). Advanced Modern Engineering Mathematics.4a ed., Inglaterra: Pearson Education, pp. 644-683. ISBN: ISBN 978-0-273-71923-6

Jiménez, César. (2007). Procesamiento digital de señales sísmicas con Matlab. Revista de Investigación de Física, 10(2): 45-50. Disponible en https://www.resea rchgate.net/publicación/280577568

Muñoz, Alejandro. (01 y 02 de setiembre del 2018). Diseño de hospitales con la norma peruana de aislamiento sísmico E.031. En: II Congreso internacional de estructuras en edificaciones. Lima, Coinesed.

Naeim, F. (1989). The Seismic Design Handbook. Estructural Engineering Series. Library of Congress Catalog Card Number 89-30616. ISBN 0-442-26922-6. 1989. Chapter 2. pp. 36-42.

Olarte, Jorge y LÓPEZ, Darwin. (2001). Sismicidad y peligrosidad sísmica en la región sur-occidental del Perú. Proyecto de investigación CISMID.

Oppenheim, Alan y WILLSKY, Alan. (2004). Señales y sistemas. $2^{\text {a }}$ ed., México: Pearson Educación, 211 pp. ISBN: 970170116X

Pérez, Mariángel. (2009). Simulación de sismos probables para la ciudad de Mérida. Tesis de posgrado. Universidad de los Andes, Venezuela. 58 pp.

Pérez, Mariangel, TORRES, Rafael, y GUADA, Carlos. (2012). Determinación de frecuencias predominantes de sismos registrados en la ciudad de Mérida, Venezuela. Boletín técnico IMME. BTI-2011-02. $11 \quad$ pp. $\quad$ Disponible en https://www.researchgate.net/publication/309477545

Red acelerográfica. Universidad Nacional de Ingeniería UPG-FIC-UNI. (15 de enero 2020). Disponible en http://www.red-acelerografica-peru.com/site/index 